\title{
Utilidad clínica de los tests de Rinne y Weber en el diagnóstico de hipoacusia
}

\section{Clinical usefulness of Rinne and Weber tests for diagnosing hearing loss}

\author{
Pedro Badía V1, Claudio Callejas C¹, Claudia González G', Pilar Gajardo 0², José Luis Anabalón B², \\ Claudio Correa $\mathrm{G}^{3}$.
}

\begin{abstract}
RESUMEN
Introducción: Los tests de Rinne y Weber son considerados una herramienta diagnóstica importante. Frente a esto nos preguntamos ¿cuál es la sensibilidad, especificidad y utilidad de los tests de Rinne y Weber?

Objetivos: 1. Estudiar la sensibilidad y especificidad de los test de Rinne y Weber en el diagnóstico de hipoacusia. 2. Comparar la sensibilidad y especificidad de dichos tests realizados por un operador habitual y un operador ocasional.

Material y método: Estudio transversal y ciego. Se incluyeron pacientes mayores de 4 años de edad con indicación de audiometría por sospecha de hipoacusia. Todos los pacientes se estudiaron con tests de Rinne y Weber y se compararon con audiometría de tonos puros.

Resultados: Se estudiaron 85 pacientes. La sensibilidad del uso combinado de los tests de Rinne y Weber, por operador ocasional, fue 76,32\% y la especificidad 88,37\%. La sensibilidad del uso combinado de los tests de Rinne y Weber por operador habitual fue $75,76 \%$ y la especificidad $79,31 \%$. En comparación al operador habitual no se observó diferencias estadísticamente significativas.

Conclusión: Los tests de Rinne y Weber representan una herramienta útil en el diagnóstico de hipoacusia.
\end{abstract}

Palabras clave: Diapasón, Rinne, Weber, audiometría, sensibilidad, especificidad.

\begin{abstract}
Introduction: Rinne and Weber tests are considered an important diagnosis tool. The question arises as to what are the tests sensitivity, specificity and usefulness.

Aim: 1. To study the sensitivity and specificity of Rinne and Weber tests in diagnosing hearing loss. 2. To compare the sensitivity and specificity of the above mentioned tests when performed by an experienced vs an occasional practitioner.

Material and method: 85 patients were studied. Rinne and Weber test combined sensibility, performed by an occasional practitioner, was $76,32 \%$, and the specificity was $79,31 \%$. There were no statistically significant differences between experienced and occasional practitioners.

Conclusion: Rinne and Weber test represent useful tools for hearing loss diagnosis.

Key words: Tuning fork, Rinne, Weber, audiometry, sensitivity, specificity.

\footnotetext{
Otorrinolaringólogo, Departamento Otorrinolaringología, Facultad de Medicina, Pontificia Universidad Católica de Chile.

Médico Cirujano, Alumno Magister Inmunología, Universidad de Chile.

Tecnólogo Médico, Departamento Otorrinolaringología, Facultad de Medicina, Pontificia Universidad Católica de Chile.
} 


\section{INTRODUCCIÓN}

Los otorrinolaringólogos estamos familiarizados con el uso de los diapasones en el estudio de la hipoacusia. A continuación revisaremos los orígenes de estos tests.

La historia de estos tests comienza con J. B. Venturi, físico, quien en 1802, en Módena, Italia, habría demostrado que la percepción de la dirección desde la cual el sonido se aproxima, se debe al hecho que un oído es estimulado por el sonido más intensamente que el otro. Luego en 1827, C. T. Tourtual, médico, en Munster, Alemania, demostró que esto también era aplicable para el sonido conducido a través de los huesos del cráneo; utilizando un reloj como fuente de sonido, encontró que la oclusión de ambos conductos auditivos externos (CAEs) aumentaba la sensación sonora en ambos oídos por igual, pero la oclusión de un solo CAE aumentaba la sensación sonora sólo en el oído ocluido, dando la impresión que el sonido provenía de ese lado. En el mismo año, C. Wheatstone, físico londinense, mientras investigaba el modo de vibración de la membrana timpánica, utilizando un diapasón, observó el mismo fenómeno descrito por Tourtual. Finalmente, en 1834, en Leipzig, Alemania, Ernst Heinrich Weber, anatomista y fisiólogo, describió una vez más el fenómeno descrito por Tourtual y Wheatstone. El quería demostrar que el sonido conducido por vía aérea es percibido por el vestíbulo y los canales semicirculares, mientras que el sonido conducido por vía ósea es percibido por la cóclea. Ninguno de estos investigadores estaba pensando en un uso clínico de sus hallazgos, y no hicieron ningún comentario al respecto. Sino que fue E. Schalmz, un otólogo, en Dresden, Alemania, que introdujo a la otología los diapasones y este test llamado posteriormente Weber ${ }^{1}$.

En 1855, A. Rinne², médico, en Gottingen, Alemania, describió el test que más tarde llevaría su nombre, como un método para comparar la conducción aérea y ósea del sonido, en un elaborado tratado que describía la fisiología de la cóclea. Él quería demostrar que en los humanos y animales terrestres, a diferencia de los que viven en el agua, la conducción del sonido a través de los huesos del cráneo era sólo un efecto colateral, inevitable, de la percepción del sonido. El mencionó una aplicación clínica de su test sólo en una nota a pie de página y nunca lo utilizó de manera sistemática en la clínica. Sin embargo algunos años antes, en 1842, Polansky habría descrito el test y su uso práctico, pero su descripción aparentemente pasó al olvido. Tuvieron que pasar más de 25 años para que Lucae en $1880^{3}$ y Schwabach ${ }^{4}$ en 1885 confirmaran la utilidad del test y se comenzará a difundir su uso $0^{5}$.

Estos tests han sido considerados, por algunos, una herramienta diagnóstica fundamental en el estudio de la hipoacusia, dada su simpleza, rapidez y bajo costo. Sin embargo con el advenimiento de tests diagnósticos más sofisticados y modernos en la audiología, han ido perdiendo su lugar, y el clínico los ha abandonado en su práctica clínica diaria.

En virtud de lo anterior nos preguntamos: ¿cuál es la sensibilidad y especificidad de los tests de Rinne y Weber? y ¿cuál es su utilidad en nuestra aproximación inicial frente a un paciente que consulta por hipoacusia?

\section{HIPÓTESIS}

El uso de los tests de Rinne y Weber en el estudio de la hipoacusia, es una herramienta útil en el diagnóstico de la hipoacusia y no requiere gran entrenamiento por parte del operador que los realiza.

\section{OBJETIVOS}

1. Estudiar la sensibilidad y especificidad de los tests de Rinne y Weber en el diagnóstico de hipoacusia.

2. Comparar la sensibilidad y especificidad de dichos tests, realizados por un operador habitual y un operador ocasional.

\section{MATERIAL Y MÉTODO}

\section{Diseño}

Se diseñó un estudio transversal, ciego.

\section{Sujetos}

Se incluyeron pacientes adultos y niños mayores de 4 años de edad, con indicación de audiometría 
por sospecha de hipoacusia, que consultaron en el Centro ambulatorio de Otorrinolaringología de la Pontificia Universidad Católica de Chile, en el período de marzo a mayo de 2006.

\section{Examinadores}

Como operadores ocasionales, fueron definidos 3 otorrinolaringólogos de 5 , 3 y 2 años de experiencia. Como operadores habituales fueron definidos 2 tecnólogos médicos audiólogos de 10 y 20 años de experiencia.

\section{Intervención}

A todos los pacientes se les realizaron tests de Rinne y Weber, con diapasón de $512 \mathrm{~Hz}$ por un operador ocasional y un operador habitual, sucesivamente. Ambos examinadores desconocían la sospecha diagnóstica y el resultado de los tests realizados por el otro examinador. Luego todos los pacientes fueron sometidos a una audiometría de tonos puros, realizada por el tecnólogo médico.

\section{Análisis}

Los datos obtenidos fueron tabulados y analizados con ExcelMR de Microsoft" ${ }^{\mathrm{MR}}$. Se estudió la sensibilidad, especificidad, valor predictivo positivo (VPP), valor predictivo negativo (VPN), cuociente de probabilidad positivo $(\mathrm{LR}+)$ y cuociente de probabilidad negativo (LR-); de cada test y del uso combinado de éstos, con respecto a la audiometría de tonos puros, para cada operador por separado. Luego se compararon dichos parámetros entre cada operador, mediante el método de distribución muestral de diferencia de proporciones.

\section{Test de Rinne}

El test de Rinne fue estudiado por oído. Su resultado se analizó con respecto a la presencia 0 ausencia de hipoacusia de conducción (diferencia ósea/ aérea $\geq 10 \mathrm{~dB}$ ) en la audiometría de tonos puros.

\section{Test de Weber}

El test de Weber fue estudiado por sujeto. Su resultado se analizó con respecto a la presencia o ausencia de hipoacusia asimétrica (diferencia entre ambos oídos $\geq 10 \mathrm{~dB}$ ) en la audiometría de tonos puros.

\section{Uso combinado de tests de Rinne y Weber}

El uso combinado de estos tests fue estudiado por sujeto. Su resultado se analizó con respecto a la presencia 0 ausencia de hipoacusia de conducción, hipoacusia mixta 0 hipoacusia sensorioneural asimétrica en la audiometría de tonos puros, lo cual fue definido como audiometría anormal.

\section{RESULTADOS}

Fueron incluidos en el estudio 85 pacientes, cuya edad promedio fue 46,5 años; su desviación estándar (DS) $\pm 21,6$ años y su rango 5 a 87 años. Su distribución por géneros fue $56 \%$ femenino y $44 \%$ masculino. El grupo femenino tuvo una edad promedio de 47,9 años $\pm 21,0 \mathrm{DS}$. El grupo masculino tuvo una edad promedio de 44,7 años $\pm 22,8$ DS.

\section{Test de Rinne por operador habitual}

En el grupo de estudio, la prevalencia de hipoacusia de conducción (HC) fue 17,8\%. La sensibilidad del test de Rinne para identificar una HC correspondió a 58,62\%. La especificidad fue $99,25 \%$. El VPP correspondió a $94,44 \%$. EI VPN correspondió a $91,72 \%$. El LR+ fue 78,55 y el LRfue 0,42 (Tabla 1).

\section{Test de Rinne por operador ocasional}

En el grupo de estudio la prevalencia de $\mathrm{HC}$ fue $19,4 \%$. La sensibilidad del test de Rinne para identificar una $\mathrm{HC}$ correspondió a 48\%. La especificidad fue $96,15 \%$. El VPP correspondió a $75 \%$. El VPN correspondió a $88,5 \%$. El LR+ fue 12,48 y el LR- fue 0,54 (Tabla 1). Al comparar estos resultados con los obtenidos por el operador habitual no se observó diferencias estadísticamente significativas.

\section{Test de Weber por operador habitual}

En el grupo de estudio la prevalencia de hipoacusia asimétrica (HA) fue $41,5 \%$. La sensibilidad del test de Weber para identificar una HA correspondió a 
Tabla 1.

\begin{tabular}{|c|c|c|}
\hline $\begin{array}{l}\text { PERADOR } \\
\text { Por Oidos }\end{array}$ & Audiom & \\
\hline RINNE & Con Gap óseo/aéreo & Sin Gap óseo/aéreo \\
\hline Negativo & 17 & 1 \\
\hline Positivo & 12 & 133 \\
\hline & SENSIBILIDAD & ESPECIFICIDAD \\
\hline & $58,62 \%$ & $99,25 \%$ \\
\hline & $40,7 \%$ a $74,5 \%$ (IC $95 \%)$ & $95,9 \%$ a $99,9 \%$ (IC $95 \%)$ \\
\hline
\end{tabular}

\begin{tabular}{|c|c|c|}
\hline \multirow{2}{*}{$\begin{array}{c}\text { PERADOR } \\
\text { Por Oidos } \\
\text { RINNE }\end{array}$} & \multicolumn{2}{|c|}{ Audiometria } \\
\hline & Con Gap óseo/aéreo & Sin Gap óseo/aéreo \\
\hline Negativo & 12 & 4 \\
\hline Positivo & 13 & 100 \\
\hline & SENSIBILIDAD & ESPECIFICIDAD \\
\hline & $48,00 \%$ & $96,15 \%$ \\
\hline & $30,0 \%$ a $66,5 \%$ (IC $95 \%)$ & $90,5 \%$ a $98.5 \%$ (IC $95 \%)$ \\
\hline
\end{tabular}

\begin{tabular}{|c|c|c|}
\cline { 3 - 3 } \multicolumn{2}{c|}{} & IC $95 \%$ \\
\hline VPP & $\mathbf{9 4 , 4 4 \%}$ & $74,2 \%$ a $99,0 \%$ \\
\hline VPN & $\mathbf{9 1 , 7 2} \%$ & $86,1 \%$ a $95,2 \%$ \\
\hline LR (+) & $\mathbf{7 8 , 5 5}$ & 10,88 a 566,93 \\
\hline LR (-) & $\mathbf{0 , 4 2}$ & 0,27 a 0,64 \\
\hline
\end{tabular}

\begin{tabular}{|c|c|c|}
\cline { 3 - 3 } \multicolumn{2}{c|}{} & IC $95 \%$ \\
\hline VPP & $\mathbf{7 5 , 0 0} \%$ & $50,5 \%$ a $89,8 \%$ \\
\hline VPN & $\mathbf{8 8 , 5 0} \%$ & $\mathbf{8 1 , 3 \%}$ a $93,2 \%$ \\
\hline LR (+) & $\mathbf{1 2 , 4 8}$ & 4,39 a 35,45 \\
\hline LR (-) & $\mathbf{0 , 5 4}$ & 0,37 a 0,80 \\
\hline
\end{tabular}

Tabla 2.

\begin{tabular}{|c|c|c|}
\hline & \multicolumn{2}{|c|}{ Audiometria } \\
\hline WEBER & Asimétrica & Simétrica \\
\hline Lateraliza & 27 & 7 \\
\hline No lateraliza & 7 & 41 \\
\hline & SENSIBILIDAD & ESPECIFICIDAD \\
\hline & $79,41 \%$ & $85,42 \%$ \\
\hline & $63.2 \%$ a $89,7 \%$ (IC $95 \%)$ & $72,8 \%$ a $92,8 \%$ (IC $95 \%)$ \\
\hline
\end{tabular}

\begin{tabular}{|c|c|c|}
\cline { 3 - 3 } \multicolumn{2}{c|}{} & IC $95 \%$ \\
\hline VPP & $\mathbf{7 9 , 4 1 \%}$ & $63,2 \%$ a $89,7 \%$ \\
\hline VPN & $\mathbf{8 5 , 4 2 \%}$ & $72,8 \%$ a $92,8 \%$ \\
\hline LR (+) & $\mathbf{5 , 4 5}$ & 2,69 a 11,03 \\
\hline LR (-) & $\mathbf{0 , 2 4}$ & 0,12 a 0,47 \\
\hline
\end{tabular}

$79,41 \%$. La especificidad fue $85,42 \%$. El VPP correspondió a $79,41 \%$. El VPN correspondió a $85,42 \%$. El LR+ fue 5,45 y el LR- fue 0,24 (Tabla 2).

\section{Test de Weber por operador ocasional}

En el grupo de estudio la prevalencia de HA fue $43,3 \%$. La sensibilidad del test de Weber para identificar una HA correspondió a $65,38 \%$. La especificidad fue $76,47 \%$. EI VPP correspondió a $68 \%$. EI VPN correspondió a 74,29\%. El LR+ fue 2,78 y el LR- fue 0,45 (Tabla 2). Al comparar estos resultados con los obtenidos por el operador habitual no se observó diferencias estadísticamente significativas.

\section{Uso combinado de tests de Rinne y Weber por operador habitual}

En el grupo de estudio la prevalencia de audiometría anormal (AA) fue 46,9\%. La sensibilidad

\begin{tabular}{|c|c|c|}
\hline & \multicolumn{2}{|c|}{ Audiometria } \\
\hline WEBER & Asimétrica & Simétrica \\
\hline Lateraliza & 17 & 8 \\
\hline No lateraliza & 9 & 26 \\
\hline & SENSIBILIDAD & ESPECIFICIDAD \\
\hline & $65,38 \%$ & $76,47 \%$ \\
\hline & $46.2 \%$ a $80,6 \%$ (IC $95 \%)$ & $60.0 \%$ a $87.6 \%$ (IC 95\%) \\
\hline
\end{tabular}

\begin{tabular}{|c|c|c|}
\cline { 3 - 3 } \multicolumn{2}{c|}{} & IC $95 \%$ \\
\hline VPP & $\mathbf{6 8 , 0 0 \%}$ & $48,4 \%$ a $82,8 \%$ \\
\hline VPN & $\mathbf{7 4 , 2 9 \%}$ & $57,9 \%$ a $85,8 \%$ \\
\hline LR (+) & $\mathbf{2 , 7 8}$ & 1,43 a 5,42 \\
\hline LR (-) & $\mathbf{0 , 4 5}$ & 0,26 a 0,80 \\
\hline
\end{tabular}

del uso combinado de los tests de Rinne y Weber para identificar una AA correspondió a $76,32 \%$. La especificidad fue $88,37 \%$. EI VPP correspondió a $85,29 \%$. EI VPN correspondió a $80,85 \%$. El LR+ fue 6,56 y el LR-fue 0,27 (Tabla 3).

\section{Uso combinado de tests de Rinne y Weber por operador ocasional}

En el grupo de estudio la prevalencia de AA fue $53,2 \%$. La sensibilidad del uso combinado de los tests de Rinne y Weber para identificar una $A A$ correspondió a $75,76 \%$. La especificidad fue $79,31 \%$. El VPP correspondió a $80,65 \%$. El VPN correspondió a $74,19 \%$. El LR+ fue 3,66 y el LRfue 0,31 (Tabla 3). Al comparar estos resultados con los obtenidos por el operador habitual no se observó diferencias estadísticamente significativas. 
Tabla 3.

\begin{tabular}{|c|c|c|}
\hline \multirow{2}{*}{ DIAPASONES } & \multicolumn{2}{|c|}{ Audiometria } \\
\hline & Anormal & Normal o HSN simétrica \\
\hline Alterado & 29 & 5 \\
\hline Normal & 9 & 38 \\
\hline & SENSIBILIDAD & ESPECIFICIDAD \\
\hline & $76,32 \%$ & $\mathbf{8 8 , 3 7 \%}$ \\
\hline & $60,8 \%$ a $87,0 \%$ (IC $95 \%)$ & $75,5 \%$ a $94,9 \%$ (IC 95\%) \\
\hline
\end{tabular}

\begin{tabular}{|c|c|c|}
\cline { 3 - 3 } \multicolumn{2}{c|}{} & IC $95 \%$ \\
\hline VPP & $\mathbf{8 5 , 2 9} \%$ & $69,9 \%$ a $93,6 \%$ \\
\hline VPN & $\mathbf{8 0 , 8 5 \%}$ & $67,5 \%$ a $89,6 \%$ \\
\hline LR (+) & $\mathbf{6 , 5 6}$ & 2,83 a 15,25 \\
\hline LR (-) & $\mathbf{0 , 2 7}$ & 0,15 a 0,48 \\
\hline
\end{tabular}

\section{DISCUSIÓN}

El test de Rinne demostró un excelente rendimiento como prueba diagnóstica en cuanto a su especificidad $(99,25 \%)$ y a su $L R+(78,55)$, realizado por un operador habitual. Esto significa que $99 \%$ de los pacientes sin $\mathrm{HC}$ tiene Rinne positivo en el oído afectado, y que un Rinne negativo aumenta 78 veces la probabilidad que el paciente tenga HC. Por lo tanto un resultado alterado hace que el diagnóstico sea muy probable y constituye un excelente test para confirmar la sospecha diagnóstica, desde el punto de vista clínico.

El test de Weber demostró un buen rendimiento como prueba diagnóstica en cuanto a su especificidad $(85,42 \%)$ y a su $L R+(5,45)$, realizado por un operador habitual. Esto significa que $85 \%$ de los pacientes con audiometría simétrica no presenta lateralización del Weber, y que una lateralización del Weber aumenta 5 veces la probabilidad que el paciente tenga una audiometría asimétrica. Por lo tanto un resultado alterado hace que el diagnóstico sea muy probable y constituye un buen test para confirmar la sospecha diagnóstica, desde el punto de vista clínico.

El uso combinado de los tests de Rinne y Weber demostró un buen rendimiento en cuanto a su especificidad $(88,37 \%)$ y a su $\operatorname{LR}+(6,56)$, realizado por un operador habitual. Esto significa que el $88 \%$ de los pacientes con audiometría normal o HSN simétrica no presenta test combinado alterado, y que un test combinado alterado aumenta 6 veces la probabilidad que el paciente tenga una audiometría anormal. Por lo tanto un

\begin{tabular}{|c|c|c|}
\hline \multirow{2}{*}{ DIAPASONES } & \multicolumn{2}{|c|}{ Audiometria } \\
\hline & Anormal & Normal o HSN simétrica \\
\hline Alterado & 25 & 6 \\
\hline \multirow[t]{4}{*}{ Normal } & 8 & 23 \\
\hline & SENSIBILIDAD & ESPECIFICIDAD \\
\hline & $\mathbf{7 5 , 7 6 \%}$ & $79,31 \%$ \\
\hline & $59,0 \%$ a $87,2 \%$ (IC $95 \%)$ & $61,6 \%$ a $90,2 \%$ (IC $95 \%)$ \\
\hline
\end{tabular}

\begin{tabular}{|c|c|c|}
\cline { 3 - 3 } \multicolumn{2}{c|}{} & IC $95 \%$ \\
\hline VPP & $\mathbf{8 0 , 6 5 \%}$ & $63,7 \%$ a $90,8 \%$ \\
\hline VPN & $\mathbf{7 4 , 1 9 \%}$ & $56,8 \%$ a $86,3 \%$ \\
\hline LR (+) & $\mathbf{3 , 6 6}$ & 1,75 a 7,66 \\
\hline LR (-) & $\mathbf{0 , 3 1}$ & 0,16 a 0,57 \\
\hline
\end{tabular}

resultado alterado hace que el diagnóstico sea muy probable y constituye un buen test para confirmar la sospecha diagnóstica, desde el punto de vista clínico.

Los tests de Rinne y Weber individual y combinadamente demostraron un rendimiento regular a moderado en cuanto a su sensibilidad ( $58 \%$ a $79 \%)$ y LR- $(0,2$ a 0,4$)$, realizados por un operador habitual. Por lo tanto un resultado normal no nos permite descartar, con seguridad, la sospecha diagnóstica, lo que implica que no sean recomendables como test de screening.

Los tests de Rinne y Weber individual y combinadamente mostraron un mejor rendimiento cuando fueron realizados por el operador habitual, en comparación al operador ocasional. Sin embargo estas diferencias no fueron estadísticamente significativas.

En la literatura se describe para estos tests una sensibilidad entre $76 \%$ y $84 \%$ \%,5,-11 y una especificidad de $85 \% 5,6$, lo cual es similar a los resultados obtenidos en nuestro estudio.

Creemos importante destacar que tanto en nuestro estudio como en publicaciones anteriores se encontró un mejor rendimiento del test de Rinne cuando es realizado por operadores experimenta$\mathrm{dos}^{5}$, si bien este hallazgo no fue estadísticamente significativo en nuestro estudio. Junto con la experiencia del operador, Burkey describe que el usar enmascaramiento durante el test de Rinne y el considerar como negativos los resultados dudosos, aumentaría la sensibilidad del examen.

Nuestros resultados permiten sospechar que el otorrinolaringólogo general ha reducido el uso de 
estos importantes tests en su práctica clínica diaria. Considerando el buen rendimiento de éstos en cuanto a especificidad y $L R(+)$ creemos que el otorrinolaringólogo debiera incluirlos en la evaluación de un paciente que consulta por hipoacusia.

\section{CONCLUSIÓN}

Nuestros resultados confirman parcialmente nuestra hipótesis ya que los tests de Rinne y Weber representan una herramienta útil en el diagnóstico de la hipoacusia tomando en cuenta su especificidad y LR+, sin embargo, a la luz de nuestros resultados, creemos que es necesario el entrenamiento y uso habitual de estos tests para mejorar su rendimiento.

Los tests de Rinne y Weber, realizados e interpretados de manera correcta, junto con la clínica del paciente, pueden no sólo minimizar los errores de otros tests audiológicos sino que además nos entregan una visión global del estado auditivo de nuestro paciente en la consulta (previo a la audiometría), lo cual ningún otro test puede realizar.

\section{BIBLIOGRAFÍA}

1. Feldman $H$. History of the tuning fork. III: On the way to quantitative pure-tone measurement. Pictures from the history of otorhinolaryngology, represented by instruments from the collection of the Ingolstadt German Medical History Museum. Laryngorhinootologie 1997; 76(7): 428-34.

2. RINNE A. Beiträge zur Physiologie des menschlichen Ohres. Vierteljahrschrift für praktische Heilkunde, herausgegeben von der medicinischen Facultät in Prag. 12.Jg. 1. Bd. 1855; 71-123, 2 Bd, 45-72: 155-6.

3. LuCAE $A$. Die bei Schwerhörigen zu beobachtende gute Perception der tiefen musikalischen Töne und die physiologische und diagnostiche Bedeutung dieser Erscheinung; nebst Section zweier bei Lebzeiten beobachteten Fälle. Arch Ohrenheilk 15. 1880; 273-85.

4. Schwabach D. Ueber den Werth des Rinne'schen Versuches für die Diagnostik der Gehörkrankheiten. Z. Ohrenheilk 14. 1885; 61148.

5. HuIzING EH. The early descriptions of the socalled tuning-fork tests of Weber, Rinne, Schwabach, and Bing. III. The development of the Schwabach and Bing tests. ORL J Otorhinolaryngol Relat Spec 1975; 37(2): 92-6.

6. BuRkey JM, LipPy WH, Schuring AG, Rizer FM. Clinical utility of the $512-\mathrm{Hz}$ Rinne tuning fork test. Am J Otol 1998; 19(1): 59-62.

7. Browning GG, Swan IR, Chew KK. Clinical role of informal tests of hearing. J Laryngol Otol 1989; 103(1): 7-11.

8. Chole RA, Cook GB. The Rinne test for conductive deafness. A critical reappraisal. Arch Otolaryngol Head Neck Surg 1988; 114(4): 399-403.

9. VikRam KB, Naseeruddin K. Combined tuning fork tests in hearing loss: explorative clinical study of the patterns. J Otolaryngol 2004; 33(4): 22734.

10. MiltenbuRg DM. The validity of tuning fork tests in diagnosing hearing loss. J Otolaryngol 1994; 23(4): 254-9.

11. Browning GG, Swan IR. Sensitivity and specificity of Rinne tuning fork test. BMJ 1988; 297(6660): 1381-2. 